\title{
THERMAL MODELS OF BIOHEAT TRANSFER EQUATIONS IN LIVING TISSUE AND THERMAL DOSE EQUIVALENCE DUE TO HYPERTHERMIA
}

\author{
Tzu-Ching ShiH ${ }^{\prime}$, Hong-Sen Kou' ${ }^{\prime}$, ChiHng-Tsung LiauH ${ }^{2}$, And WIN-Li Lin $^{3}$ \\ ${ }^{1}$ Department of Mechanical Engineering, Tatung University, Taipei \\ ${ }^{2}$ Department of Mechanical Engineering, Kun Shan University of Technology, Tainan \\ ${ }^{3}$ Institute of Biomedical Engineering, National Taiwan University, Taipei, Taiwan
}

\begin{abstract}
This review focuses both on the basic formulations of bioheat equation in the living tissue and on the determination of thermal dose during thermal therapy. The temperature distributions inside the heated tissues, generally controlled by heating modalities, are obtained by solving the bioheat transfer equation. However, the major criticism for the Pennes' model focused on the assumption that the heat transfer by blood flow occurs in a non-directional, heat sink- or source-like term. Several bioheat transfer models have been introduced to compare their convective and perfusive effects in vascular tissues. The present review also elucidates thermal dose equivalence that represents the extent of thermal damage or destruction of tissue in the clinical treatment of tumor with local hyperthermia. In addition, this study uses the porous medium concept to describe the heat transfer in the living tissue with the directional effect of blood flow, and the polynomial expression of thermal dose in terms of the curve fitting of the experimental isosurvival curve data by Dewey et al. Results show that the values of factor $R$ is a function of the heating temperature instead of the two different constants suggested by Sapareto and Dewey.
\end{abstract}

Biomed Eng Appl Basis Comm, 2002 (April); 14: 86-96.

Keywords: Bioheat transfer, Bioheat equation, Thermal dose

\section{INTRODUCTION}

During hyperthermia/thermal therapy, bioheat transfer processes in living tissue are often significantly affected by the blood flow of the vascular networks on treatment distributions. Even although circulating blood only comprises about $10 \%$ of the total body volume, the convective and perfusive effects of blood flow play an essential role and may contribute up to $90 \%$ of all heat transport in living tissues [1].

Received: March 27, 2002; accepted: April 10, 2002 Correspondence: Hong-Sen Kou, Ph.D., Professor Department of Mechanical Engineering, Tatung University No. 40, Chung-Shan North Rd., Sec. 3, Taipei, 104 Taiwan

Email:hskou@me.ttu.edu.tw
The most difficult problem of estimating heat transfer in perfused biological tissues is to model the convection effect of blood circulation. In 1948, Pennes first proposed quantifying heat transfer effects in perfused tissue of the human forearm by the pure scalar term such heat source/sink. This term is directly proportional to the blood perfusion rate and the difference between the global arterial temperature and the tissue/venous temperature. Pennes made the underlying assumptions that the blood perfusion is homogeneous in tissue and all heat transfer occurs in the capillary beds. However, many investigators questioned the assumptions and found them not valid for some the physiology conditions. As the heat transfer interaction between the vessels and the surrounding tissues depends on the vascular architecture and the properties of blood and the surrounding tissues, many investigators have developed alternative models for describing the 
bioheat transfer instead of the Pennes model [2-3]. The successful clinical tumor treatment with local hyperthermia requires the detailed temperature distribution in tumor and normal tissue. Until now, the practical mathematical model for describing the heat transfer in living tissues during hyperthermia/therapy is not yet develo ped. In addition, Mooibroek et al. proposed the three major problems in the formulation of thermal model [4]. The first problem is to identify the heat exchange between the vessel structures. The second problem is to assess whether these structures are accessible for a continuum formulation. The third problem is to cope with the dynamic behavior of tissueblood heat exchange, i.e., the physiological response at elevated tissue temperatures. Furthermore, it is well known that the thermal dose is a function of both heating temperature and time. This study is aimed to explore the features of existing thermal models and to describe the determination of thermal dose equivalence.

\section{FORMULATION OF BIO-HEAT TRANSFER MODELS}

Nine thermal models have been reviewed in the formulation of the heat transfer in living tissues. The related formulae appeared in each model are briefly written for the convenience of advanced discussion.

\subsection{The Pennes' Model}

The first quantitative mathematical relationship that described the heat transfer between blood and tissue is presented by Pennes in 1948. The Pennes' model [5] for describing the energy balance of tissue metabolism and blood perfusion in a living tissue is designed originally for predicting temperature fields in the human forearm. For simplicity of the modeling analysis, Pennes made three assumptions that the rate of heat production by tissue, the volume flow of blood per unit volume of tissue per second and the tissue specific thermal conductivity were both considered uniform throughout the forearm. Although the Pennes bioheat model is developed based on the experimental analysis of the human foreman, it can be used to quantitatively calculate the rate of heat transfer in any perfused tissues. It has been adopted by many authors who have developed mathematical models of heat transfer in living tissues so that it becomes well known as the bioheat transfer equation. According to the human forearm temperature measurement results, Pennes quantitatively analyzed the brachial arterial blood and tissue temperatures and further evaluated the applicability of heat flow theory to the forearm in terms of local rate of tissue heat production and volume flow of blood. The principal theoretical contribution of Pennes shows that the rate of heat transfer between blood and tissue is proportional to the product of the volumetric perfusion rate and the difference between the arterial blood temperature and the local tissue temperature. As Pennes was not certain of the extent of thermal equilibrium between capillary blood and surrounding tissue, he presumed the physical conditions of the capillary circulation almost complete equilibrium. Furthermore he induced the equilibration constant to discuss its influence on the mean experimental temperature curves and the theoretical curves. According to his suggestion that the arterial blood temperature is considered uniform throughout the tissue, the thermal energy balance for perfused tissue is expressed in the following form

$$
\rho_{t} c_{t} \frac{\partial T}{\partial t}=\nabla \cdot\left(k_{t} \nabla T\right)-W_{b} c_{b}\left(T-T_{a}\right)+q_{m}
$$

where $\rho$ is the density; $c$ is the specific heat; $k$ is the thermal conductivity with the subscripts $t$ and $b$ referring to tissue and blood domain, respectively; $T$ is the temperature; $q_{m}$ heat generations due to metabolism; $W_{b}$ is the blood perfusion rate; and $T_{a}$ is the arterial blood temperature. Note that $q_{m}$ is $0.0001 \mathrm{cal} /\left(\mathrm{cm}^{3} \cdot \mathrm{s}\right)$ and $W_{b}$ ranges from 0.0002 to $0.0005 \mathrm{~g} /\left(\mathrm{cm}^{3} \cdot \mathrm{s}\right)$. The four terms in Equation (1) are intended to represent thermal energy storage, thermal energy diffusion, convective energy (i.e., energy perfusion of solids by liquids, primarily by blood), and metabolic heat generation. In Equation (1), the blood perfusion is modeled as a uniform heat sink/source term. It neglects the direction of local capillary perfusion and the influence of larger vessels in proximity to the local tissue volume elements. The solution of BHTE depends on the specific initial and boundary conditions and may be obtained by well-known mathematical methods.

\subsection{The Mitchell and Myers Model}

Mitchell and Myers [6] described the thermal energy balance for the extremity of an animal with the countercurrent heat exchange mechanism. The contercurrent heat exchange pattern can be divided into three individual energy components under the energy conservation law. Under the assumptions including (a) the temperatures of the arterial and venous flows varied with distance in the flow direction only, (b) the thermal conductances between artery and vein, artery and environment, and vein and environment were independent of distance along the limb, (c) the mass flow rates of the arterial and venous flows were equal and constant with distance, (d) the thermal energy generated due to metabolism was small relative to the heat transfer terms, (e) the limb was in a steady state; temperatures and flow rates were not changing with time, ( $f$ ) the thermal properties of the blood and tissue were constant, they investigated the effect of countercurrent heat exchange between an artery and a vein, and the heat exchange of each vessel and its surrounding tissue. Their equations of modeling 
Their equations of modeling are defined as:

Arterial flow energy equation is:

$$
\dot{m} c \frac{d t_{a}}{d x}+\left(U A^{\prime}\right)_{i}\left(t_{a}-t_{v}\right)+\left(U A^{\prime}\right)_{a}\left(t_{a}-t_{\infty}\right)=0
$$

Venous flow energy equation is:

$$
-\dot{m} c \frac{d t_{v}}{d x}+\left(U A^{\prime}\right)_{i}\left(t_{v}-t_{a}\right)+\left(U A^{\prime}\right)_{v}\left(t_{v}-t_{\infty}\right)=0
$$

The boundary conditions on the temperature are:

$$
x=0: t_{a}=t_{0} ; x=L: t_{a}=t_{v},
$$

where the thermal conductance, $U$, the heat transfer area per length, $A^{\prime}$, and the temperature difference causing the heat flow $(\Delta t)$, the temperatures $t$ with the subscripts $a$ and $v$ refer to the artery and vein. Their found that the countercurrent effect becomes more significant as the blood flow rate decreases.

\subsection{The Keller and Seiler Model}

Keller and Seiler [7] focused on the heat transfer phenomena of the subcutaneous region beneath of the skin. The region of interest was divided into two regions: an isothermal core and a peripheral region where the temperature varied only in a direction normal to the skin surface. Assuming that heat conduction in the $\mathrm{x}$ direction was negligible compared with the convection effect of the flowing blood, they not only incorporated the countercurrent heat exchange as reported by Mitchell and Myers [6] but also added an energy conservation equation for the surrounding tissue that coupled with the artery and vein equations.

$$
\begin{aligned}
& k \frac{d^{2} T}{d x^{2}}+(h a+c \dot{g})\left(T_{a}-T\right)+h a\left(T_{v}-T\right)+q_{m}=0 \\
& {\left[\left(\dot{m}_{a}\right)_{0}-\int_{0}^{x} \dot{g} d x\right] c \frac{d T_{a}}{d x}+h a\left(T_{a}-T\right)=0} \\
& {\left[\left(\dot{m}_{a}\right)_{0}-\int_{0}^{x} \dot{g} d x\right] c \frac{d T_{v}}{d x}+(h a+c \dot{g})\left(T-T_{v}\right)=0}
\end{aligned}
$$

with the following boundary conditions

$$
x=0, T=T_{a}=T_{b} ; x=\delta, T=T_{v}=T_{\text {s }}
$$

where $k$ is the thermal conductivity of the tissue, $x$ is the length variable in the direction normal to surface, $h$ is the average heat transfer coefficient from vessels to surrounding tissues, a is the average area for heat transfer per unit volume, $\mathrm{c}$ is the heat capacity, $\dot{g}$ is the capillary perfusion rate, $\dot{m}$ is the blood flow rate, $\delta$ is the thickness of tissue layer, and the subscripts a, $\mathrm{b}, \mathrm{v}$, and $\mathrm{s}$ represent artery, isothermal core, vein, and skin, respectively. In addition, Keller and Seiler found that the increasing heat transfer in subcutaneous region was induced by the capillary perfusion rate and by the decreasing arterial precooling.

\subsection{The Wulff Model}

Wulff [8] examined on the perfusion term of the Pennes model and further pointed out the three incorrect things in the Pennes bioheat transfer equation.
Firstly, the local (i.e. the energy storage, the diffusion, and generation terms) and global (i.e. the convection term) control systems were in Pennes mode. Secondly, three media with three temperatures occupied simultaneously the same point of space. Thirdly, he claimed that the convective effect originating from the net blood flux within the tissue and the blood flow contribution must be modeled by a directional convection term $\rho_{b} c_{b} U_{h} \cdot \nabla T$ rather than the scalar perfusion term suggested by Pennes [5].

$\rho_{t} c_{t} \frac{\partial T}{\partial t}=\nabla \cdot\left(k_{t} \nabla T\right)-\rho_{b} c_{b} U_{h} \cdot \nabla T+q_{m}$,

where $U_{h}$ is the local mean apparent blood velocity associated with the enthalpy flux.

\subsection{The Chen and Holmes Model}

Applying the control volume concept, Chen and Holmes [9] presented that the perfused tissue volume treated as a combination of the solid subvolume and the vascular subvolume occupied by blood. They addressed the equilibration length of a single to evaluate the equilibration length for different generations of blood vessels and found the equilibration length was very sensitive to vessel radius. Furthermore, they also found the blood temperatures of the precapillary arterioles, capillaries, and venules were essentially equal to the solid tissue temperatures. In other words, the equilibration between blood and tissue has already occurred before the capillary, not only in the capillary previously suggested by Pennes [5]. It is indicate that the energy equations of the blood and tissue must be described individually by two different equations. In addition, Chen and Holmes suggested that the perfusion heating term in the continuum formulation should be based on the blood flow rates and the blood temperatures. Finally, they also considered the contribution of the blood flow in the microvasculature to local blood perfusion velocity and the effective thermal conductivity. The Chen and Holmes equation can be expressed in the form:

$$
\begin{aligned}
\rho_{,} c_{1} \frac{\partial T}{\partial t}= & \nabla \cdot\left(k_{t} \nabla T\right)+\rho_{b} c_{b} W^{\cdot}\left(T_{a}^{\cdot}-T\right)-\rho_{b} c_{b} u_{p} \cdot \nabla T \\
& +\nabla \cdot k_{p} \nabla T+q_{m}
\end{aligned}
$$

where $W^{*}$ is the perfusion rate; $u_{p}$ represents the net volume flux vector of the blood permeating a unit area of the control surface, $k_{p}$ is the perfusion conductivity, subscripts $t$ and $b$ are referred to tissue and blood, respectively.

\subsection{The Weinbaum, Jiji and Lemons Model}

Weinbaum, Jiji and Lemons [10-11] utilized the tissue clearance method to analyze the tissue heat transfer. The method was serially sectioned parallel to 
the skin surface to determine the detailed variation of the vascular geometry as a function of tissue depth. From the ultrastuctural observations and the highspatial resolution temperature measurements of a rabbit thigh, they addressed the three layer heat transfer models due to the different characteristics of anatomical structures. Starting form the skin surface to the deep tissue, the three layer simplified conceptual models are the cutaneous vein near surface, the countercurrent exchange, and the vessel-tissue cylinder. Except for the simple models in the foregoing, Weinbaum et al. looked at the phenomena of an axial decay due to radial diffusion in the blood vessels and the surrounding tissue. Furthermore, they defined the thermal relaxation length of an isolated vessel for blood flow in different size vessels. The thermal relaxation length is different from the equilibration length defined by Chen and Holmes [9]. However, Weinbaum et al. and Chen and Holmes also get the same important conclusion that the thermal equilibration between blood and tissue is in the arterioles and venules, of course, in all capillary networks. The three layer conceptual models of WJL and their mathematical formulations are as follows:

\section{(a) Deep Tissue Layer}

The deep tissue layer begins at the initial branching of the terminal arteriole-venous arcades where the countercurrent vessels are typically $300 \mu \mathrm{m}$ in diameter and have a spacing of about $1 \mathrm{~cm}$ between pairs. The deep tissue layer equations are as follows:

$$
\begin{aligned}
& \rho_{b} c_{b} a^{2} \bar{u} \frac{d}{d s}\left(T_{v}-T_{v}\right)=2 \rho_{b} c_{s} a y\left(T_{a}-T_{v}\right)+\frac{\bar{K}}{2}-\frac{d}{d s}\left|R^{2} \frac{d}{d s}\left(T_{o}+T_{v}\right)\right|, \\
& \rho_{h} c_{b} a^{2} \bar{u} \frac{d}{d s}\left(T_{a}+T_{v}\right)=-4 K\left(T_{a}-T_{\nu}\right) / \cosh ^{\prime}\left(\frac{l^{2}}{2 a^{2}}-1\right)
\end{aligned}
$$

Equations (6a) and (6b) can be solved simultaneously for $T_{a}(s)$ and $T_{v}(s)$ with the boundary conditions

$$
T_{a}(0)=T_{0} ; \quad T_{v}(0)=T_{r},
$$

where $a$ is the vessel radius, $\bar{u}$ is the mean blood flow velocity, $s$ is the coordinate along artery-vein pair axis, $g$ is the volumetric blood bleed-off per unit artery surface area leaving arterial or entering venous flow, $T_{a}$ is the artery bulk temperature, $T_{v}$ is the vein bulk temperature, $K$ is the thermal conductivity of the tissue in deep layer and intermediate layer, $l$ is the distance between centers of artery-vein pair in deep tissue layer, and $T_{0}$ is the arterial blood temperature entering the deep tissue layer. The venous return temperalure $T_{r}$ is unknown and must be determined as part of the solution to the coupled three layers model.

\section{(b) Intermediate Layer}

In the intermediate layer, the paired vessels eventually separate and form a roughly periodic arrangement of equally spaced transverse terminal vessels. The transverse terminal artery is represented as a line source and the transverse terminal vein as a line sink. The conservation of energy in the arterial and venous tissue cylinders, expressed in dimensionless form, yiclds

$$
\begin{aligned}
& \beta^{2} \frac{\partial^{2} \tilde{\theta}_{a}}{\partial \tilde{x}^{2}}+\frac{\partial^{2} \tilde{\theta}_{a}}{\partial \tilde{r}^{2}}+(1-v) \frac{1}{\tilde{r}} \frac{\partial \tilde{\theta}_{a}}{\partial \tilde{r}}=-i \\
& \beta^{2} \frac{\partial^{2} \tilde{\theta}_{v}}{\partial \tilde{x}^{2}}+\frac{\partial^{2} \tilde{\theta}_{v}}{\partial \tilde{r}^{2}}+(1+v) \frac{1}{\tilde{r}} \frac{\partial \tilde{\theta}}{\partial \tilde{r}}=-\lambda
\end{aligned}
$$

and the boundary conditions are

$$
\begin{aligned}
& \partial \tilde{\theta}_{u}(0, \tilde{x}) / \partial r=0 \\
& \tilde{\theta}_{u}(\tilde{r}, 0)=F(\tilde{r}) \\
& \tilde{\theta}_{u}(\tilde{r}, 1)=\widetilde{\phi}_{0} \\
& \partial \tilde{\theta}_{v}(0, \tilde{x}) / \partial r=0 \\
& \tilde{\theta}_{v}(\tilde{r}, 0)=G(\tilde{r}) \\
& \tilde{\theta}_{v}(\tilde{r}, 1)=\tilde{\phi}_{0} \\
& \tilde{\theta}_{a}(1, \tilde{x})=\tilde{\theta}_{v}(1, \tilde{x}), \\
& \partial \widetilde{\theta}_{u}(1, \tilde{x}) / \partial \tilde{r}=\partial \tilde{\theta}_{v}(1, \tilde{x}) / \partial \widetilde{r}
\end{aligned}
$$

where $F(\tilde{r}), G(\tilde{r})$, and $\tilde{\phi}_{0}$ account for the thermal coupling between the intermediate layer and the two neighboring regions. They are unknown and must be determined by matching the solutions to the system of equations for the three-layer model.

\section{(c)Cutaneous Layer}

The temperature distribution in this layer is assumed to be one-dimensional, varying with the distance $y$ normal to the skin. This layer is subdivided into two regions, and inner region, $0<y<y_{1}$, which contains the cutaneous plexus and an outer region, $y_{1}<y<y_{0}$, which contains only thermally insigniticant smaller vessels of the arterial and venous plexus. In the first layer, $0<y<y_{1}$, the one-dimensional conservation of energy for this region is:

$$
\frac{d^{2} \tilde{\phi}_{1}}{d \tilde{y}^{2}}+W_{b}^{2}\left(\tilde{\phi}_{b}-\tilde{\phi}_{1}\right)=0
$$

with

$$
W_{h}^{2}=2 \pi \rho_{h} c_{b} a_{c} n_{c} g_{c} y_{0}^{2} / K_{1}
$$

where $\widetilde{\phi}_{1}$ is the dimensionless temperature distribution in the inner region of cutaneous layer, $K_{1}$ is the 
mean thermal conductivity of the blood and tissue in the region, $q_{c}$ is the rate of energy transfer from the blood in the cutaneous plexus to the tissue per unit volume. In the second region, $y_{1}<y<y_{0}$, energy transfer is assumed to be one-dimensional, governed by the conduction equation

$$
\frac{d^{2} \widetilde{\phi}_{2}}{d \tilde{y}^{2}}=0 \text {, }
$$

where the $\widetilde{\phi}_{2}$ is the dimensionless temperature distribution in the outer region of cutaneous layer. The boundary conditions for equations (6dl) and (6d 3$)$ are

$$
\begin{aligned}
& \tilde{\phi}_{1}(0)=\widetilde{\phi}_{0} \\
& \widetilde{\phi}_{1}\left(\tilde{y}_{1}\right)=\widetilde{\phi}_{2}\left(\tilde{y}_{1}\right) \\
& d \widetilde{\phi}_{1}\left(\widetilde{y}_{1}\right) / d \tilde{y}=d \widetilde{\phi}_{2}\left(\tilde{y}_{1}\right) / d \tilde{y} \\
& \int_{0}^{1} \frac{\partial \tilde{\theta}_{a}(\tilde{r}, 1)}{\partial \tilde{x}} \widetilde{r} d \widetilde{r}+\int_{0}^{1} \frac{\partial \tilde{\theta}_{v}(\widetilde{r}, 1)}{\partial \tilde{x}} \widetilde{r} d \tilde{r}=\xi_{0} \frac{d \tilde{\phi}_{1}(0)}{\partial \tilde{y}}
\end{aligned}
$$

where $\xi_{0}=x_{0} / y_{0}$.

Weinbaum, Jiji and Lemons found that in cutaneous tissue layer conduction is the dominant heat transfer mechanism and that blood perfusion plays an insignificant role, and in the deep tissue layer conuntercurrent heat exchange is the dominant heat transfer mechanism. In addition, they suggested that in bioheat equation the blood perfusion term must replace by a simplified description of the incomplete countercurrent exchange mechanism.

\subsection{The Weinbaum and Jiji Model}

Obviously, the previous WJL mathematical model is too complex and difficult to solving for the coupling boundary value problem of the three layers so that Weinbaum and Jiji [12] discuss in some limiting cases. Weinbaum and Jiji focused on the effect of countercurrent exchange and capillary bleed off on local tissue heat transfer and derived an enhancement conductivity term expressed in terms of the local vascular geometry and flow velocity due to the significant countercurrent convection. Weinbaum and Jiji described the local blood-tissue heat exchange with the countercurrent vessels as follows:

$$
\begin{aligned}
& \overline{\rho c} \frac{\partial \theta}{\partial t}-\nabla \cdot k \nabla \theta=-\pi \rho_{h} c_{h} n a^{2} u \frac{d}{d s}\left(T_{a}-T_{v}\right) \\
& +2 \pi \rho_{b} c_{b} \operatorname{nag}\left(T_{a}-T_{v}\right)+q_{m},
\end{aligned}
$$

where $\overline{\rho c}$ is the average blood-tissue capacity, $\theta$ is the mean temperature, $n$ is the number density of vessel pairs crossing surface of the control volume per unit area, $u$ is the average blood velocity in countercurrent artery or vein, $a$ is the artery radius, and $g$ is the volumetric blood bleed-off rate per unit vessel surface area leaving arterial flow or entering venous flow;
$T_{u}$ and $T_{v}$ are described by separated energy equations for the arterial and venous blood in the countercurrent vessels. In the WJL model, there are three equations that could be solved simultaneously as described in [11]. Here, Weinbaum and Jiji made a simpler description in which local arterial-venous temperature difference $T_{a}-T_{v}$ and its directional derivative in the equation (7a) can be related to the local tissue temperature and its derivatives. In the first term on the right-hand side of equation (7a), it presents the dominant contribution of the countercurrent blood-tissue heat exchange. It gives rise to the effective conductivity tensor.

The coupling between the local countercurrent arterial-venous temperature difference and the mean tissue temperature gradient in the direction of the vessel axis is

$$
\pi \rho_{b} c_{b} a^{2} u \frac{d \theta}{d s}=-\sigma k\left(T_{a}-T_{v}\right)
$$

where $\sigma$ is the shape factor. Substituting equation (7b) in (7a) and introducing the dimensionless Peclet number and considering the opposing blood flows in the countercurrent vessels carrying the memory of the local temperature in all the three directions in terms of tensor conductivity, yields

$\overline{\rho c} \frac{\partial \theta}{\partial t}-\frac{\partial}{\partial x_{i}}\left[\left(k_{i j}\right)_{e f} \frac{\partial \theta}{\partial x}\right]=-\frac{\pi n a^{2} k_{b}^{2}}{4 \sigma k} P e^{2} l_{1}, \frac{\partial l_{1}}{\partial x_{1}} \frac{\partial \theta}{\partial x_{1}}+q_{m}$,

where $x_{l}$ is the coordinate axis $i=1,2,3, k_{y}$ is the thermal conductivity of anisotropic material, $l$, and $l$, are the direction cosines, and $P e$ number is defined by

$$
P e=\operatorname{Pr} \operatorname{Re}=2 p_{b} c_{b} a u / k_{b}
$$

For the simple one-dimensional case where the blood vessels and the temperature gradient are in the same direction, the effective tissue conductivity can be reduced to the scalar equation as given below

$$
k_{e f f}=k\left(1+\frac{\pi^{2}}{4 \sigma k^{2}} n a^{2} k_{b}^{2} P e^{2}\right)
$$

The $W J$ equation provides a simple expression for determining the effect of the Reynolds number on heat transfer, as shown in the above equation (7e). Weinbaum and Jiji found that the heat exchange of the countercurrent vessels in diameter less than $0.05 \mathrm{~mm}$ is negligible and the only significant countercurrent vessels have a significant enhancement due to directed perfusion.

\subsection{The Weinbaum, Xu, Zhu, and Ekpene Model}

Weinbaum, Xu, Zhu, and Ekpene [21] used the basic unit of skeletal muscle tissue concept suggested by Myrhage and Eriksson [20] to describe the effect of 
blood perfusion on local tissue heat transfer. Weinbaum et al. believed that the total heat transfer released in the muscle tissue cylinder surrounding secondary vessels is determined by calculating the temperature difference between the countercurrent artery and vein. Accounting for the net heat loss to the tissue from the incomplete countercurrent exchange, they also used the simplified description of the microvascular architecture of the Weinbaum and Jiji equation [12]. They used the solutions of velocity components in the axial and radial directions $u_{a}$ and $v_{a}$ in the artery which are given by

$$
\begin{aligned}
& u_{a}=2 u_{o v}\left(1-\frac{\dot{z}}{\dot{L}^{\bullet}}\right)\left(1-\frac{r_{a}^{.2}}{a_{a}^{.2}}\right)
\end{aligned}
$$

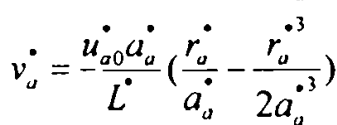

The dimensionless parameters and variables are defined as:

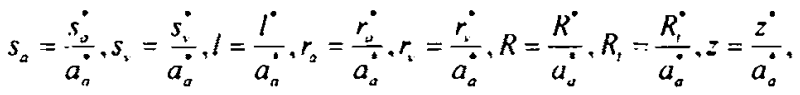

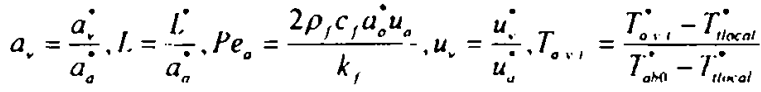

Here the subscripts $a$, and $v$ refer to the s artery and vein, respectively, and asterisks denote dimensional variables. $a$ is the vessel radius and $s$ is the vessel eccentricity. The governing equations for the artery and vein reduce to

$\frac{1}{r_{a}} \frac{\partial}{\partial r_{a}}\left(r_{a} \frac{\partial T_{o}}{\partial r_{a}}\right)+\frac{1}{r_{a}^{2}} \frac{\partial^{2} T_{a}}{\partial \phi_{a}^{2}}=P e_{a}\left(1-\frac{z}{l}\right)\left(1-r_{a}^{2}\right) \frac{d T_{o b}}{d z}, \quad r_{o} \leq 1$

$\frac{1}{r_{v}} \frac{\partial}{\partial r_{v}}\left(r_{v} \frac{\partial T_{v}}{\partial r_{v}}\right)+\frac{1}{r_{v}^{2}} \frac{\partial^{2} T_{v}}{\partial \phi_{v}^{2}}=u_{v} P e_{a}\left(1-\frac{z}{L}\right)\left(1-\frac{r_{v}^{2}}{a_{v}^{2}}\right) \frac{d T_{v 0}}{d z}, \quad r_{v} \leq a_{v}$

The tissue temperature field in the cross-sectional plane can be regarded as the temperature field generated by a line heat source-sink pair representing the secondary vessels in a porous media with convection. Considering the porous medium concept, Weinbaum et al. introduced the relationships that the velocity field $U$ satisfies Darcy's law and represents the flow through the terminal vessels and their subsequent branching. The potential flow field can be approximated by the flow between an equal line source and sink located along the axes of the secondary vessels, which are $0.05-0.1 \mathrm{~mm}$ in diameter.

$$
\nabla^{2} T_{t}=P e_{a} \tilde{U} \cdot \nabla T_{t}, \quad R \leq R_{\imath}
$$

For resting conditions, the governing equation for the tissue region reduces to

$$
\frac{1}{R} \frac{\partial}{\partial R}\left(R \frac{\partial T_{i}}{\partial R}\right)+\frac{1}{R^{2}} \frac{\partial^{2} T_{i}}{\partial \phi^{2}}=0, R \leq R_{t}, r_{a}>1, r_{v}>a_{v} .
$$

Since the thermal equilibration in the terminal vessels is almost instantaneous, the arterial and venous blood at the wall of the secondary vessels is the same as the tissue temperature and the latter termperature is the same as the blood temperature in the terminal vessels when it returns or exits in the secondary vessel pair.

$$
T_{a}=T_{1}, r_{a}=1 ; T_{v}=T_{1}, r_{a}=a_{v}
$$

For the energy flux both diffusive and convective fluxes must be considered,

$$
\begin{aligned}
& \frac{\partial T_{a}}{\partial r_{a}}+\left.P e_{a} \frac{v_{a}^{\cdot}}{u_{a 0}^{\bullet}} T_{a}\right|_{r_{u}=1}=\frac{\partial T_{1}}{\partial r_{a}}+P e_{a} \frac{\tilde{U}}{u_{u 0}^{\bullet}} T_{r_{r_{a}=1}} \\
& \frac{\partial T_{v}}{\partial r_{v}}+\left.P e_{a} \frac{v_{v}^{*}}{u_{a 0}^{*}} T_{v}\right|_{v=a_{v}}=\frac{\partial T_{1}}{\partial r_{v}}+\left.P e_{u} \frac{\widetilde{U}^{*}}{u_{u}^{*}} T_{1}\right|_{v_{v}=u_{v}}
\end{aligned}
$$

However, from continuity $v_{a}^{*}=\tilde{U}^{\bullet}$ and $v_{v}^{*}=\tilde{U}^{\bullet}$ at $r_{u}=1$ and $r_{v}=a_{v}$, respectively, and $T_{a}=T_{1}, T_{v}=T_{1}$. Thus, Equations $(8 \mathrm{~h})$ and $(8 \mathrm{i})$ reduce to the forms as:

$$
\begin{aligned}
& \frac{\partial T_{v}}{\partial r_{a}}=\frac{\partial T_{1}}{\partial r_{a}}, r_{a}=1 \\
& \frac{\partial T_{v}}{\partial r_{v}}=\frac{\partial T_{1}}{\partial r_{v}}, r_{v}=a_{v}
\end{aligned}
$$

Finally, the boundary condition on the cylindrical surface of the tissue is given by

$$
T_{t}=0 \text {, at } R=R \text {, }
$$

Note that the boundary condition is essential to the analysis since it requires the blood to equilibrate to the local tissue temperature in the small vessels. In equations $(8 \mathrm{c})$ and $(8 \mathrm{~d}), T_{a h}$ and $T_{v b}$ are artery and vein bulk temperatures, respectively, and defined as

$$
\begin{aligned}
& T_{a b}=\frac{2}{\pi} \int_{-\pi}^{\pi} \int_{0}^{1} T_{a}\left(1-r_{a}^{2}\right) r_{u} d r_{v} d \phi_{a} \\
& T_{v b}=\frac{2}{\pi a_{v}^{2}} \int_{-\pi}^{\pi} \int_{0}^{a_{v}} T_{v}\left(1-\frac{r_{v}^{2}}{a_{v}^{2}}\right) r_{v} d r_{v} d \phi_{v}
\end{aligned}
$$

From theoretical analysis, Weinbaum et al. found the higher flow rate introduces the longer equilibration length. On the other hand, the lower blood flow in the vessels contributes little to the axial thermal interaction so that the axial thermal equilibrium is almost unchanged. In addition, they also found that the countercurrent heat exchange becomes more significant than the capillary bleed off when the countercurrent vessels are near and the ratio of the tissue cylinder radius to the artery radius is much larger than unity.

\subsection{The Porous Model}


Shih et al. [22] proposed that heat transfer in living tissues, including blood flow in microvascular bed, could be modeled by solving a conjugate problem with the porous medium concept. This model contains a solid sub-domain for the tissue and a liquid subdomain for the blood that couple throughout their common boundary conditions. For the tumor tissue, neglecting the metabolic heat production term, it is possible to simplify the Pennes bioheat transfer equation shown in Equation (9a). The energy transport equation of blood flow is used here as shown in Equation (9b).

$$
\begin{aligned}
& \rho_{1} c_{1} \frac{\partial T_{t}}{\partial t}=k_{1}\left(\frac{\partial^{2} T_{t}}{\partial x^{2}}+\frac{\partial^{2} T_{1}}{\partial y^{2}}+\frac{\partial^{2} T_{t}}{\partial z^{2}}\right)-W_{b} c_{b}\left(T_{t}-T_{b}\right) \\
& +q_{1}(x, y, z, t) \text {, } \\
& \rho c_{b}\left(\frac{\partial T_{b}}{\partial t}+u \frac{\partial T_{b}}{\partial x}+v \frac{\partial T_{b}}{\partial y}+w \frac{\partial T_{b}}{\partial z}\right) \\
& =k_{b}\left(\frac{\partial^{2} T_{b}}{\partial x^{2}}+\frac{\partial^{2} T_{b}}{\partial y^{2}}+\frac{\partial^{2} T_{b}}{\partial z^{2}}\right)+W_{b} c_{b}\left(T_{t}-T_{b}\right)+q_{b}(x, y, z, t)
\end{aligned}
$$

where $\rho$ is the density, $c$ is the specific heat, $k$ is the thermal conductivity, $T$ is the temperature, and $q(x, y, z, t)$ is the absorbed power deposition density with the subscripts $t$ and $b$ referring to tissue and blood domain, respectively; $W_{b}$ is the blood perfusion rate. If considering the thermal equilibrium conditions of blood vessels in treatment tissues, we obtain the three different cases: Case1) Blood temperature and tissue temperature are not the same (i.e. no thermal equilibration). The full governing equations must include the individual equations (9a) and (9b). Note that the two equations have a blood-tissue coupled term as $W_{b} c_{b}\left(T_{t}-T_{b}\right)$ to describe the heat exchange between blood and tissue. This term will be vanished when the blood and tissue temperatures are the same. Case 2) If considering the region of tissue near the large vessel where the blood vessel is greater than $0.5 \mathrm{~mm}$ in diameter and $T_{b}$ is the constant blood temperature, the full governing equations are reduced to the traditional Penns bioheat equation when $T_{b}=T_{a}$ is the arterial blood temperature/core temperature. In other words, the governing equation is exactly equation (9a) only. Case 3) Blood and tissue temperatures are the same. The full governing equation just is one equation only. On the other hand, blood and tissue temperatures are at a state of local thermal equilibrium (such as in the pre-capillary arterioles, the capillary network, and the post-capillary venules presented by Chen et al. [9] and Weinbaum et al. [10]). The volumetric averaging of the energy conservation principle is achieved by combining and rearranging equations (9a) and (9b) with the porous structure regarded as a homogeneous medium to get Equation (9c).

$$
\begin{aligned}
& {\left[\phi \rho_{b} c_{b}+(1-\phi) \rho_{l} c_{l}\right] \frac{\partial T}{\partial t}+\phi \rho_{b} c_{h}\left[u \frac{\partial T}{\partial x}+v \frac{\partial T}{\partial y}+w \frac{\partial T}{\partial z}\right]} \\
& =\left[\phi k_{b}+(1-\phi) k_{l}\right]\left(\frac{\partial^{2} T}{\partial x^{2}}+\frac{\partial^{2} T}{\partial y^{2}}+\frac{\partial^{2} T}{\partial z^{2}}\right)-(1-\phi) q_{1}+\phi q_{b}
\end{aligned}
$$

The porosity, $\phi$, is defined as the ratio of blood volume to the total (solid tissue + liquid blood) volume. In equation $(9 \mathrm{c})$, herein there are two characteristic features including not only the convective term (instead of a non-directional blood perfusion term) as likely suggested by Wulff [8] but also the porous medium property (advancing the control volume concept and introducing by the porous porosity) as likely proposed by Chen and Holmes [9].

\section{DETERMINATION OF THERMAL DOSE}

\subsection{Temperature Cytotoxic Effect}

Heat can change the properties of proteins in the cells (i.e., cytotoxic effects). When heat-induced denaturation of proteins takes place in different cellular compartments, the denatured proteins tend to aggregate [23-25]. Lepock et al. used differential scanning calorimetry to assay thermal transitions that might be responsible for cell death and other responses to hyperthermia. They found that the onset temperature of denaturation of Chinese hamster lung V79 cells is at $38.7^{\circ} \mathrm{C}$. For this reason, hyperthermia is currently being studied as a modality for cancer therapies [25]. The quantification of cell-killing is a function of the temperature and the duration of the exposure of treatments. Dewey found that the effect of heat shock (hyperthermia) on mammalian cells is to block proliferation [26]. Exposure of mammalian cells to temperatures above $41^{\circ} \mathrm{C}$ leads to a gradual loss of the cells' productive capacity (reproductive cell death)[2728]. Survival curves are generated for mammalian cells in culture (Chinese hamster ovary cells) heated at different temperatures for varying lengths of time [29]. Dewey et al. found that when the temperature is lower to about $42.5^{\circ} \mathrm{C}$, the thermal resistance of a pig kidney cell appears as the survival curves flatten after the treatment duration of about 300 minutes. Bromer et al. suggested that the thermotolerance of human hematopoietic precursor cells at developed $42^{\circ} \mathrm{C}$ [31]. In addition, other investigators had observed the thermotolerance of $\mathrm{CHO}$ and HeLa cells at 43.5$45.5^{\circ} \mathrm{C}[32-33]$. However, it is usually applied as an additive therapy to enhance the effect of conventional radio- or chemotherapy [34-35]. 


\subsection{Arrhenuis Relationship and Thermal Isoef- fect Dose}

Quantitatively the relationship between the rate of chemical reactions and its absolute temperature is determined by the Arrhenius equation. At higher temperatures, the probability that two molecules will collide is higher. This higher collision rate results in a higher kinetic energy, which has an effect on the activation energy of the reaction [29]. The thermodynamics of heat inactivation can be described as follow:

$$
1 / D_{0}=A \exp [-\Delta H / 2 T]
$$

where $A$ is effectively a constant; and $T$ is the absolute temperature, and

$$
\ln \left(1 / D_{0}\right)=\ln A-(\Delta H / 2)(1 / T)
$$

which gives the Arrhenius plot when $\log 1 / D_{0}$ is plotted on the ordinate and $1 / T$ is plotted on the abscissa. Equation (10a) shows how $D_{0}$ changes with temperature; therefore, the relative decrease $R$ in $D_{0}$ for a one degree increase in temperature is:

$R=\left(D_{0}\right.$ for $\left.\mathrm{T}+1\right) /\left(D_{0}\right.$ for $\left.\mathrm{T}\right)=\exp [-\Delta H / 2 T(T+1)]$

For $\mathrm{CHO}$ cells, $\Delta H=141 \mathrm{kcal} / \mathrm{mole}$, and from $43^{\circ} \mathrm{C}$ to $46^{\circ} \mathrm{C}$,

$$
R=\exp [-0.690]=0.50
$$

The slope of the Arrhenius plot for this experiment gives an activation energy of $148 \mathrm{kcal} / \mathrm{mole}$ and a ratio of 0.48 for the relative decrease in $D_{0}$ for a $1^{\circ}$ rise in temperature over the temperature range 43$46.5^{\circ} \mathrm{C}$. Note that the value of about 0.5 also pertains to comparisons at survival values in the first decade before the curves become exponential. In other words, if the temperature is lowered to $1^{\circ} \mathrm{C}$, the treatment time for an isoeffect must be increased by a factor of 2 .

\subsection{Thermal Dose Defined by Temperature and Duration}

The effect of hyperthermia treatment depends on the temperature and the duration of heating [36]. If a constant temperature could be maintained, then, the duration of heating would be a reasonable way of expressing thermal dose with units of time. However, for practical purposes, a constant temperature is not maintained, so it is necessary to find a method of relating a treatment to an equivalent time at a specified reference temperature. The relative biological effect of a heating treatment can be estimated by calculating the thermal dose acquired over the course of the thermal treatment. The thermal isoeffect dose method uses the Arrhenius relationship to convert any temperature history to an equivalent magnitude of minutes at a standard temperature, such as $43^{\circ} \mathrm{C}$ (Sapareto and Dewey, 1984) [36].

The thermal dose or thermal equivalent minutes at $43^{\circ} \mathrm{C}\left(E M_{43}\right)$ defined by Sapareto and Dewey can describe the extent of thermal damage or destruction of tissues, which is a function of the temperature elevation and time. The Sapareto-Dewey expression is,

$$
E M_{43}=\int_{t_{0}}^{t} R^{43-T} d t,
$$

Table 1. Treatment time and $\mathbf{R}$ for each isosurvival fraction at different treatment temperature

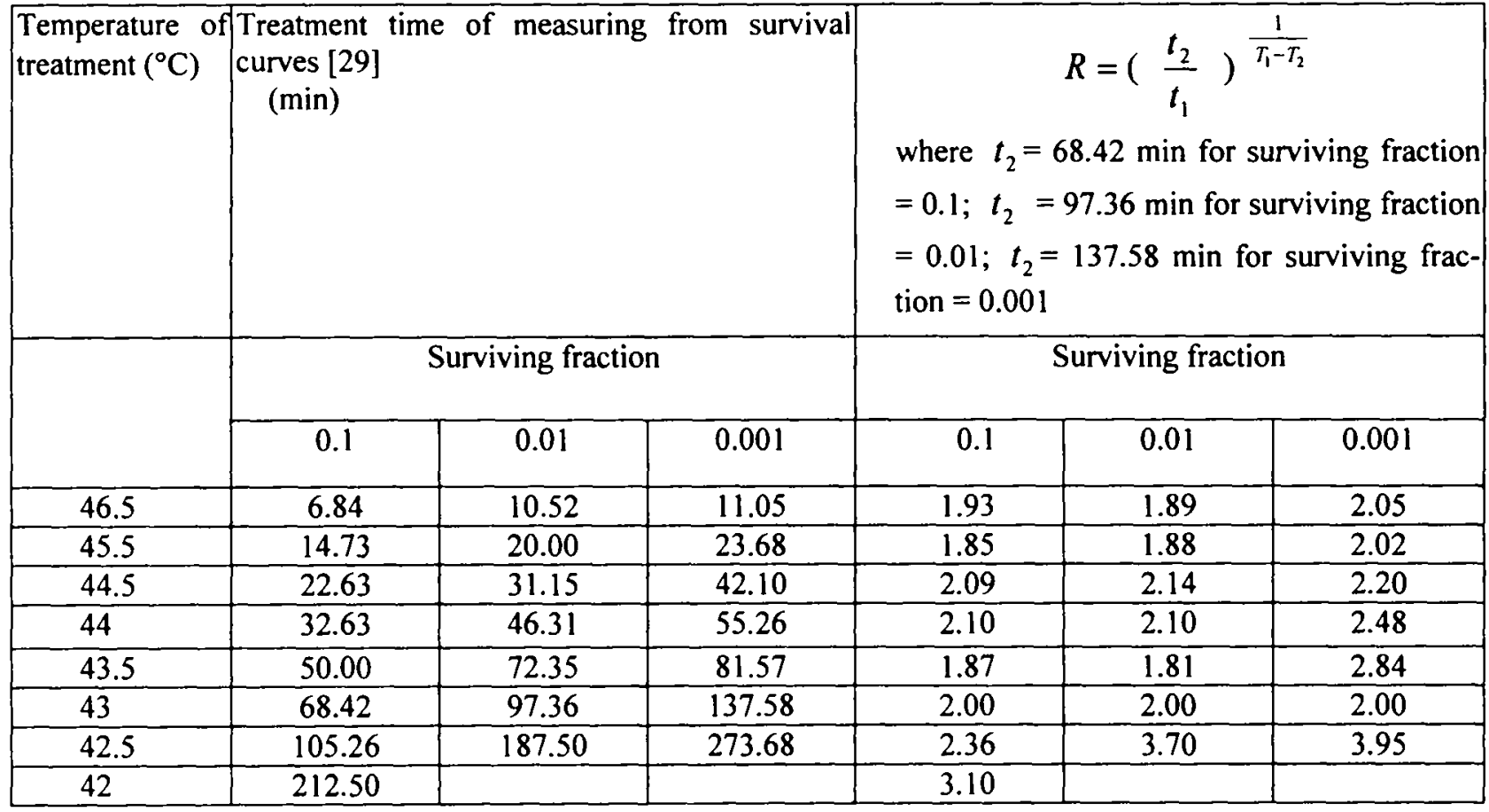




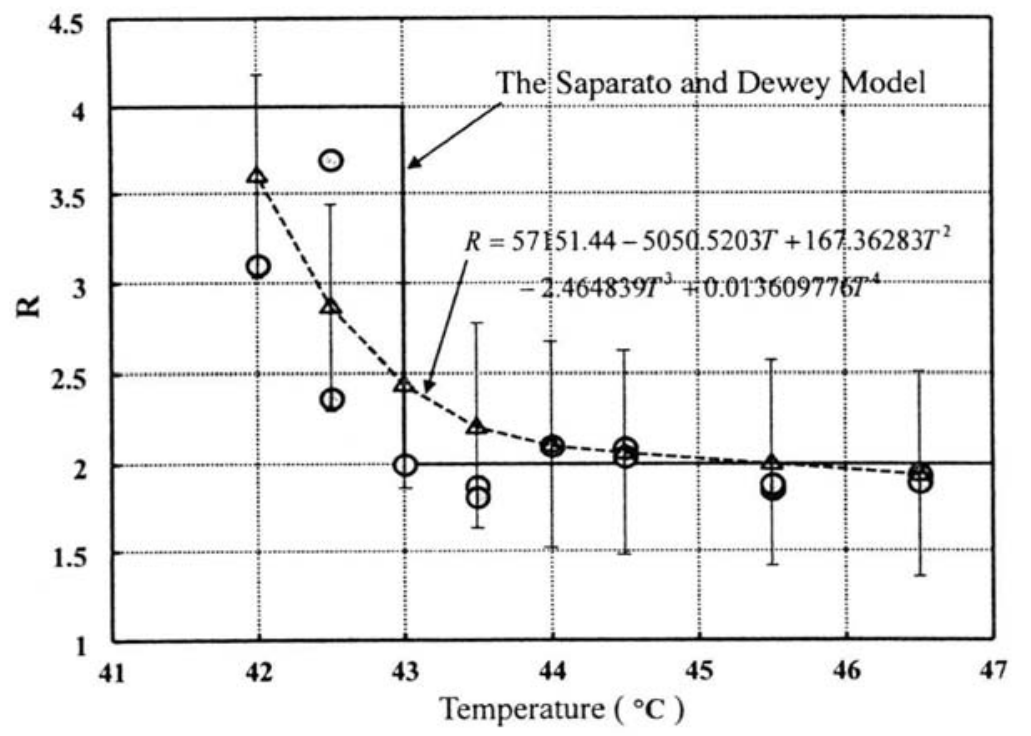

Fig. 1. The illustration of the relationship between the modified exponential form of $R$ and data calculated by equation (13) of measuring survival curves for asynchronous Chinese hamster ovary cells heated at different temperatures. The error bars represent the standard error of the

where $\mathrm{R}=0.5$ for $\mathrm{T} \geq 43^{\circ} \mathrm{C}$ and $\mathrm{R}=0.25$ for $37^{\circ} \mathrm{C}<\mathrm{T}<$ $43^{\circ} \mathrm{C}, \mathrm{T}$ is the temperature, $t_{0}$ is the initial heating time, $t_{f}$ is the final heating time. Equation (11) provides a useful method to calculate the accumulated dose at a reference temperature under a variety of heating distributions, including those temperature histories that cannot be easily described mathematically during thermal treatment. The threshold for necrosis ranges from $E M_{43}=25$ to $240 \mathrm{~min}$ for brain and muscle tissues, respectively. Based on the transient achieved transient temperature distributions obtained by solving the heat transfer equation in a living tissue, and the accumulating thermal dose can theoretically be used to predict the efficiency of thermal therapy and then prevent the lesion of the surrounding normal tissue. Many factors such as blood flow, thermal adaptation, heating schemes, and combinations with irradiation or other modalities, are influencing the effectiveness of thermal therapy. It is clear that an adequate temperature must be delivered for an appropriate duration to the target tumor volume in order to achieve optimal therapeutic results. However, non-homogeneous temperature distributions are frequently encountered, particularly in larger tissues.

\subsection{The Polynomial Expression of Thermal Dose}

The dose-modifying factors are applied to the mathematical model. The relationship of the slope survival curve changes with temperatures has been described as follows [29]:

$$
t_{2}=t_{1} R^{T_{1}-T_{2}}
$$

where $t_{2}$ is the equivalent time at $43^{\circ} \mathrm{C}, t_{1}$ is the exposure time during hyperthermia treatments, $T_{1}$ is the temperature during hyperthermia treatments, $T_{2}$ is the reference temperature $\left(43^{\circ} \mathrm{C}\right), R$ can be calculated as a function of the inactivation energy of the critical rate-limiting molecules in the cell causing cell lethality. Rearranging equation (12), it can be expressed as:

$$
R=\left(\frac{t_{2}}{t_{1}}\right)^{\frac{1}{T_{1}-T_{2}}} .
$$

Equation (13) provides a means of relating treatments to a given tissue with different temperatures and heating durations. From the survival curves for asynchronous hamster cells heated at different temperatures for varying lengths of time, there are three different cases of survival as shown in Table 1.

Applying the isosurvival data for asynchronous hamster cells heated at each temperature into equation (13) and using the curve fitting, as shown in Fig. 1, the $4^{\text {th }}$ degree polynomial form of the $R$ can be obtained as follow:

$$
\begin{aligned}
R= & 57151.44-5050.5203 T+167.36283 T^{2} \\
& -2.464839 T^{3}+0.013609776 T^{4}
\end{aligned}
$$

Finally, the thermal dose calculator can be expressed as follows:

$$
\begin{aligned}
E M_{43}= & \int_{t_{s}}^{t_{f}}\left[57151.44-5050.5203 T+167.36283 T^{2}\right. \\
& \left.-2.464839 T^{3}+0.013609776 T^{4}\right]^{T-43} d t .
\end{aligned}
$$




\section{SUMMARY}

No general thermal model has been developed which is suitable for any body site, especially for the description of convection heat transfer by blood flow in perfused tissues. More accurate formulation of thermal dose to predict therapeutic effect on tumor mass and thermal model to analyze the heat transfer in tissues based on the detailed anatomy need to be developed. Without considering of the large vascular architecture, the bioheat transfer equation of Pennes model still has acceptable results to predict the transient temperature due to its simplicity and flexibility. Except for the range below $43^{\circ} \mathrm{C}$, the Sapareto and Dewey thermal dose model describes closely the survival data. In addition, the more accurate formulation of thermal dose model is based on the more precise intratumor temperature distribution during thermal therapy.

\section{REFERENCES}

1. Guyton AC: Textbook of medical physiology. Saunders, Philadelphia 1986; 206-336.

2. Baish JW: Heat transfer mechanisms in vascular tisssues: A model comparison. ASME Trans.: J. Biomech. Eng. 1986; 108: 324-331.

3. Arkin $\mathrm{H}, \mathrm{Xu} \mathrm{LX}$, and Holmes KR: Recent developments in modeling heat transfer in blood perfused tissues. IEEE Trans. Biomed. Eng. 1994; 41: 97-105.

4. Mooibroek J, Crezee J, and Lagendijk JJW: Basic of thermal models: Thermoradiotherapy and Thermochemotherapy, Berlin, Spring, 1995:425-

5. Mannes HH: Analysis of tissue and anterial blood temperatures in the resting forearm. J. Appl. Physiol. 1948; 1: 93-122.

6. Mitchell JW and Myers GE: An analytical model of the countercurrent heat exchange phenomena. Biophys. J. 1968; 8:897-911.

7. Keller KH and Seiler L: An analysis of peripheral heat transfer in man. J. Appl. Physiol. 1971; 30: 779-786.

8. Wulff $W$ : The energy conservation equation for living tissue. IEEE Trans. Biomed. Eng. 1974; BME21: 494-495.

9. Chen MM, and Holmes KR: Microvascular contributions in tissue heat transfer. Ann. N.Y. Acad. Sci. 1980; 335:137-151.

10. Weinbaum S, Jiji LM, and Lemons DE: Theory and experiment for the effects of vascular microstruvture on surface tissue heat transfer -Part I: Anatomical foundation and model conceptualization. ASME Trans.: J. Biomech. Eng. 1984; 106:321-330.
11. Jiji LM, Weinbaum S, and Lemons DE: Theory and experiment for the effect of vascular microstructure on surface tissue heat transfer -Part II: Model formulation and solution. ASME Trans.: J. Biomech. Eng. 1984; 106: 331-341.

12. Weinbaum S and Jiji LM: A new simplified bioheat equation for the effect of blood flow on local average tissue temperature. ASME Trans.: J. Biomech. Eng. 1985; 107: 131-139.

13. Wissler EH: Comments on the new bioheat equation proposed by Weinbaum and Jiji. ASME Trans.: J. Biomech. Eng. 1987; 109:226-233.

14.Zhu M, Weinbaum S, Jiji LM, and Lemons DE: On the generalization of the Weinbaum-Jiji bioheat equation to microvessels of unequal size; the relation between the near field and local average tissue temperatures. ASME Trans.: J. Biomech. Eng. 1988; 110: 74-81.

15. Charny CK, Weinbaum S, and Levin RL: An evaluation of the Weinbaum-Jiji bioheat equation for normal and hyperthermia conditions. ASME Trans.: J. Biomech. Eng. 1990; 112: 80-87.

16. Weinbaum S, Jiji LM, and Lemons DE: The bleed off perfusion term in the Weinbaum-Jiji bioheat equation. ASME Trans.: J. Biomech. Eng. 1992; 114: 539-542.

17. Valvano JW, Nho S, and Anderson GT: Analysis of the Weinbaum-Jiji model of blood flow in the canine kidney cortex for self-heated thermistors. ASME Trans.: J. Biomech. Eng. 1994; 119: 201207.

18. Baish JW: Formulation of a statistical model of heat transfer in perfused tissue. ASME Trans.: J. Biomech. Eng. 1994; 116: 521-527.

19.Zhu L, Lemons DE, and Weinbaum S: A new approach for predication the enhancement in the effective of perfused muscle tissue due to hyperthermia. Ann. Biomed. Eng. 1995; 23:1-12.

20. Myrhage R, and Eriksson E: Arrangement of the vascular bed in different types of skeletal muscles. Prog. Appl. Microcirc. 1984; 5:1-14.

21. Weinbaum S, Xu LX, Zhu L, and Ekpene A: A new fundamental bioheat equation for muscle tissue: Part I--blood perfusion term. ASME Trans.: J. Biomech. Eng. 1997; 119: 278-288.

22. Tzu-Ching Shih, Hong-Sen Kou, and Win-Li Lin: Effect of effective tissue conductivity on thermal dose distributions of living tissue with directional blood flow during thermal therapy. Int. Comm. Heat Mass Transfer 2002;29:1 15-126.

23. Lepock JR, Cheng KH, Al-Qysi $\mathrm{H}$, et al.: Thermotropic lipid and protein trasitions in Chinese hamster lung cell membranes. Canadian J. Biochem. Cell Biol. 1983; 61: 421-427.

24.Lepock JR, Frey HE, Rodahl M, et al.: Thermal analysis of CHL V79 cells using differential scanning calorimetry: implications for hyperthermic 
cell killing and the heat-shock response. J. Cell Physiol. 1988; 137: 14-24.

25. Hall EJ and Roizin-Towle L: Biological effects of heat. Cancer Res. Suppl. 1984; 44: 4708s-4713s.

26. Dewey WC: The search for critical cellular targets damaged by heat. Radiat. Res. 1989; 120:191-204.

27. Sapareto SA, Hopwood LE, and Dewey WC: Combined effects of $\mathrm{X}$-irradiation and hyperthermia on CHO cells for various temperatures and orders of application. Radiat. Res. 1978; 73: 221-233.

28. Sapareto SA, Hopwood LE, Dewey WC, et al.: Effects of hyperthermia on survival and progression of Chinese hamster ovary cells. Cancer Res. 1978; 38: 393-400.

29. Dewey WC, Hopwood LE, Sapareto SA, et al:: Cellular response to combinations of hyperthermia and radiation. Radiat. Biol. 1977; 123: 463-474.

30. Westra A and Dewey WC: Variation in sensitivity to heat shock during the cell-cycle of Chinese hamster cells in vitro. Int. J. Radiat. Biol. 1971; 19: 467-477.
31. Bromer RH, Mitchell JB, and Soares N: Response of human hematopoietic precursor cells (CFUc) to hyperthermia and radiation. Cancer Res. 1982; 42: 1261-1265.

32. Gerner EW, Boone R., Connor WG, Hicks JA, and Boone ML: A transient thermotolerant survival response produced by single thermal doses in $\mathrm{HeLa}$ cells. Cancer Res. 1976; 36: 1035-1040.

33. Henle $\mathrm{KJ}$ and Leeper $\mathrm{DB}$ : Interaction of hyperthermia and radiation in $\mathrm{CHO}$ cells. Radiat. Res. 1976; 66: 505-518.

34.Emami B, Mittal BM, and Sapareto S: Sequencing of the total course of hyperthermia and irradiation. Cancer Res. Suppl. 1984; 44: 4731s-4732s.

35. Perez CA and Sapareto SA: Thermal dose expression in clinical hyperthermia and correlation with tumor response/control. Caner Res. Suppl. 1984; 44 4818s-4825s.

36. Saprareto SA and Dewey WC: Thermal dose determination in cancer therapy. Int. J. Radiat. Biol. 1984; 10: 787-800. 